\title{
Randomized controlled trial of brief cognitive behavioral intervention for depression and anxiety symptoms preoperatively in patients undergoing coronary artery bypass graft surgery
}

\author{
Tam K. Dao, PhD, ${ }^{\text {a,b }}$ Nagy A. Youssef, MD, ${ }^{\text {c,d,e }}$ Mary Armsworth, ${ }^{\text {a }}$ Emily Wear, ${ }^{\text {a }}$ \\ Katina N. Papathopoulos, ${ }^{a}$ and Raja Gopaldas, $\mathrm{MD}^{\mathrm{f}, \mathrm{g}}$
}

\begin{abstract}
Objective: The goal of this study was to examine the feasibility, acceptability, and efficacy of a brief, tailored cognitive-behavioral intervention for patients with symptoms of preoperative depression or anxiety before undergoing a coronary artery bypass graft $(\mathrm{CABG})$ operation.
\end{abstract}

\begin{abstract}
Methods: Patients were recruited from a university teaching hospital between February 2007 and May 2009. Patients were randomly assigned to receive treatment as usual (TAU) or a cognitive behavioral therapy (CBT) intervention called Managing Anxiety and Depression using Education and Skills (MADES). A total of 100 subjects were randomized into the study. Length of hospital stay was assessed with a 1-way analysis of variance. Depression, anxiety, and quality of life were assessed with mixed-model repeated measures analyses.
\end{abstract}

\begin{abstract}
Results: Overall, the intervention was feasible, and patients had a positive impression of the MADES. Patients in the TAU group stayed longer in the hospital than did those in the MADES group ( 7.9 days \pm 2.6 vs 9.2 days \pm $3.5 ; P=.049$ ). Depressive symptoms increased at time of hospital discharge for the TAU group, whereas the MADES group had a decrease in depressive symptoms at the time of discharge. Quality of life and anxiety symptoms improved in both groups at 3 to 4 weeks of follow-up. However, the MADES group had greater improvements than did the TAU group.

Conclusions: This study demonstrated that brief, tailored CBT targeting preoperative depression and anxiety is both feasible and acceptable for patients undergoing CABG surgery. Most important, this intervention improved depressive and anxiety symptoms, as well as quality of life. Moreover, it reduced in-hospital length of stay. This study found that a cognitive-behavioral intervention for patients undergoing CABG surgery for symptoms of preoperative depression/anxiety is both feasible and acceptable. Most important, this intervention improved depressive and anxiety symptoms, as well as quality of life. It also reduced in-hospital length of stay. (J Thorac Cardiovasc Surg 2011;142:e109-15)
\end{abstract}

Approximately 469,000 coronary artery bypass graft (CABG) operations are performed annually in the United States, making it one of the most commonly performed invasive operations. ${ }^{1}$ Patients for whom CABG surgery is recommended may incur significant psychological distress as a result of both coronary artery disease (CAD) and the procedure itself. To date, multiple longitudinal and cross-

\footnotetext{
From the University of Houston, ${ }^{\text {a }}$ Houston, Tex; Baylor College of Medicine and the Department of Psychiatry and Behavioral Sciences, ${ }^{b}$ Houston, Tex; Department of Psychiatry and Behavioral Sciences, ${ }^{c}$ Duke University Medical Center, Durham, NC; Mid-Atlantic Mental Illness Research, Education, and Clinical Center, ${ }^{\text {d Dur- }}$ ham Veterans Affairs Medical Center, Durham, NC; University of South Alabama, ${ }^{\mathrm{e}}$ College of Medicine, Mobile, Ala; Division of Cardiothoracic Surgery, ${ }^{\mathrm{f}}$ University of Missouri -Columbia School of Medicine, Columbia, Mo; and Harry S. Truman Veterans Affairs Medical Center, ${ }^{\mathrm{g}}$ Columbia, Mo.

This study was supported by Mental Illness Research, Education, and Clinical Center. Disclosures: Authors have nothing to disclose with regard to commercial support.

Received for publication Sept 29, 2010; revisions received Jan 10, 2011; accepted for publication Feb 9, 2011; available ahead of print May 30, 2011.

Address for reprints: Tam K. Dao, PhD, University of Houston, Baylor College of

Medicine, Houston, TX 77204 (E-mail: tdao@mail.coe.uh.edu).

$0022-5223 / \$ 36.00$

Copyright (c) 2011 by The American Association for Thoracic Surgery

doi:10.1016/j.jtcvs.2011.02.046
}

sectional studies have examined the association between psychological functioning and CABG surgery outcomes, particularly with depression. ${ }^{2-4} \mathrm{~A}$ review of the literature using MEDLINE and PsychINFO produced over 100 studies investigating this relationship. These studies provide evidence for the following: depression occurs in $18 \%$ to $60 \%$ of patients with heart disease; it predicts postoperative complications, longer physical and emotional recovery, poorer quality of life, and increased rates of cardiac events and mortality. ${ }^{2-6}$

Despite the important contribution of these studies, the principal unanswered questions in this field remain primarily treatment related. ${ }^{7,8}$ Although only a handful of clinical trials examined the benefits of treating depression or anxiety in CABG patients postoperatively, ${ }^{7,9-11}$ no published clinical trials have specifically examined the impact of cognitive-behavioral approaches in treating depression or anxiety on CABG patients preoperatively. A review of the literature indicated that only 2 clinical trials have examined whether treating depressed heart patients for depression postoperatively has any impact 


$$
\begin{aligned}
& \text { Abbreviations and Acronyms } \\
& \text { BDI-II = Beck Depression Inventory-II } \\
& \text { CABG = coronary artery bypass graft } \\
& \text { CAD = coronary artery disease } \\
& \text { CBT }=\text { cognitive behavioral therapy } \\
& \text { MADES = } \text { Managing Anxiety and Depression } \\
& \text { using Education and Skills } \\
& \text { MINI = } \text { Mini International } \\
& \text { Neuropsychological Interview } \\
& \text { SF-12 }= 12 \text {-item Short Form Health Survey } \\
& \text { STAI }= \text { State-Trait Anxiety Inventory } \\
& \text { TAU }= \text { treatment as usual }
\end{aligned}
$$

on cardiovascular outcomes. SADHART (Sertraline AntiDepressant Heart Attack Trial) was a small safety study that found a trend toward reduced cardiovascular mortality and morbidity with selective serotonin reuptake inhibitors but was too small and short to draw useful conclusions. $^{12}$ The ENRICHD (Enhancing Recovery in Coronary Heart Disease) trial found that cognitive behavioral therapy (CBT) after myocardial infarction had a modest effect on depression ${ }^{13}$ but did not affect cardiac events such as nonfatal infarction, death from any cause, and cardiac death.

The current study examined the feasibility of a brief, tailored CBT intervention, Managing Anxiety and Depression using Education and Skills (MADES), for treating patients with CAD and symptoms of depression or anxiety who have been scheduled to undergo CABG surgery. Furthermore, the study assessed the impact of this intervention on depression/anxiety symptoms and in-hospital length of stay.

\section{METHODS \\ Participants}

Institutional review board approval was obtained from the hospital facility as well as from the investigators' University Human Subject's Committee. This study was conducted in accordance with the Declaration of Helsinki. Patients were recruited from a Veterans Affairs hospital between February 2007 and May 2009. Patients signed informed consent forms and were enrolled if they had clinically significant symptoms of depression and/or anxiety according to the Beck Depression Inventory-II (BDI-II; score $\geq 14$ ) or the State-Trait Anxiety Inventory (STAI; score $\geq 40$ on either the State or Trait Scale), respectively ${ }^{14,15}$ These patients also had a CAD diagnosis and were scheduled to undergo a first-time CABG operation without concomitant valve procedures. Subjects were excluded if they had any of the following: serious medical illness other than CAD, psychiatric instability (eg, suicidality), schizophrenia, bipolar disorder, active alcoholism or substance abuse, severe cognitive impairment, noncardiac illnesses with a poor 1-year prognosis, previous exposure to cognitive-behavioral treatment within the past year, or receiving ongoing psychotherapeutic services. Of note, current use of psychotropic medications, such as an antidepressant medication, was not an exclusion criterion, so long as the patient has been taking the medication for at least 4 weeks. Recruitment of subjects consisted of having a cardiothoracic surgeon and team (registered nurses, nurse practitioners, and physician assistants) evaluate all potential patients who were referred to undergo CABG surgery. Those who were identified as candidates for the CABG and who had been scheduled to undergo the operation in the next 4 to 10 days were further screened by the principal investigator or a trained member on the study team. Patients were told that the screening was to evaluate their mood and that they may qualify for a research project that will be explained to them if they meet the study's eligibility criteria. Eligible patients had four 60-minute sessions with the therapist for treatment of depression and anxiety symptoms. Figure 1 presents the flow of subjects through the study. A total of 513 patients were screened for eligibility from February 2007 to May 2009. Of those, 413 subjects were excluded owing to being ineligible for the study. Thus, a total of 100 subjects were randomized into the study. The sample consisted of mostly men $(\mathrm{n}=76 ; 78 \%)$ with an average age of 63.6 years $(\mathrm{SD}=15.3)$. There was no significant difference between subjects assigned to the MADES treatment and those assigned to treatment as usual (TAU) on any of the demographic variables. The majority of subjects were white $(\mathrm{n}=76$, or $76 \%), 20 \%$ were African American, and $4 \%$ were Hispanic American. In terms of marital status, $22 \%$ were single, $58 \%$ were married, $5 \%$ were separated, and the remainder were divorced or widowed. In regard to education level, $72 \%$ had completed high school, $6 \%$ had greater than 12 years of education, and the remainder had less than 12 years of education. Regarding household income, $22 \%$ were in the range of $\$ 20,000$ or less, $52 \%$ were in the range of $\$ 20,001$ to $\$ 40,000,21 \%$ were in the range of $\$ 40,001$ to $\$ 60,000$, and $5 \%$ were in the range of $\$ 45,001$ or greater.

On the basis of the Mini International Neuropsychological Interview (MINI), ${ }^{16}$ subjects had a variety of comorbid psychiatric diagnoses that included panic disorder $(\mathrm{n}=38)$, obsessive-compulsive personality disorder $(n=2)$, social phobia $(n=11)$, posttraumatic stress disorder $(n=51)$, bulimia nervosa $(\mathrm{n}=1)$, and adult attention-deficit/hyperactive disorder $(\mathrm{n}=2)$. There were no significant differences between the groups at pretreatment on the measures of depression and anxiety (see Table 3). Twenty patients enrolled in the study were receiving psychotropic medications. $\chi^{2}$ analysis revealed that the proportion of patients receiving medications did not differ significantly between groups, $\chi^{2}(1,97)=1.11$, $P=.291$

\section{Procedures}

Referred patients with a CAD diagnosis who have been scheduled to undergo a CABG in the next week completed a consent for screening that consisted of the BDI-II, STAI-Trait, 12-item Short Form Health Survey (SF-12), and the MINI. Individuals who scored 14 or greater on the BDI-II or 40 or greater on the STAI were randomly assigned using a random numbers table to receive the TAU or a brief form of CBT.

Baseline measures were completed at least 7 days before the scheduled operation. Follow-up assessments were completed at the end of treatment (posttest 1) and when patients came back for their 3- to 4-week follow-up appointment with their cardiac team (posttest 2). Thus, the BDI-II and the STAI-Trait inventories were administered in the last session of the treatment while patients were still in the hospital. At the 3- to 4-week followup, the BDI-II, STAI-Trait, and the SF-12 were administered. In addition, patients in the intervention group completed a questionnaire about the credibility of the intervention at the end of the first session. At the end of the fourth session, patients in the intervention group completed a measure of treatment satisfaction.

\section{Measures}

Screening and outcome measures. The BDI-II and the STAITrait were used to assess levels of anxiety and depression. ${ }^{14,15}$ MINI was used to determine psychiatric diagnoses. ${ }^{16}$ Length of hospital stay, the BDI-II, and the STAI-Trait scales scores were the primary outcome 


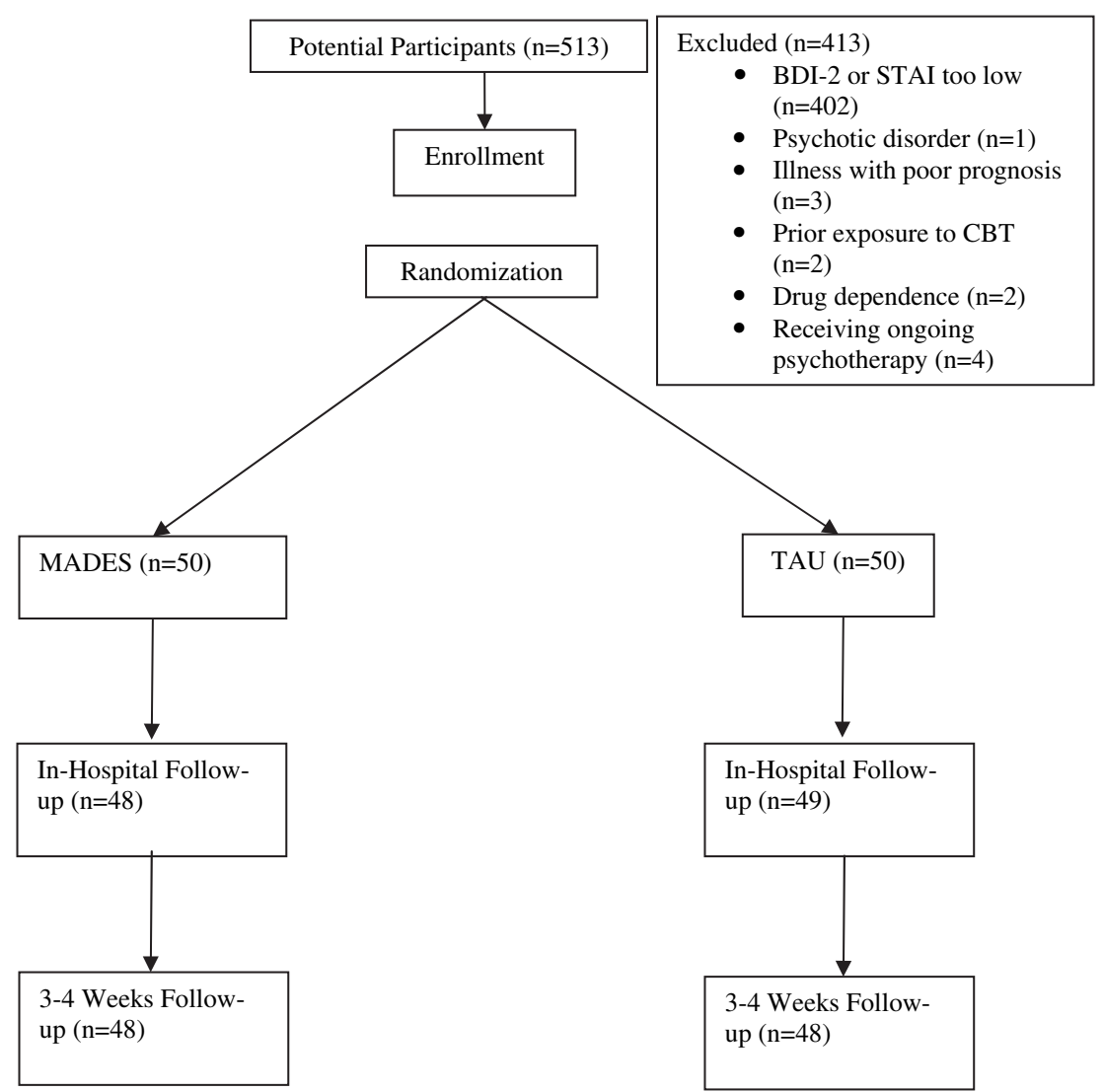

FIGURE 1. Participant enrollment, assignment, and follow-up. BDI-2, Beck Depression Inventory-II; STAI, State-Trait Anxiety Inventory; CBT, cognitive behavioral therapy; MADES, Managing Anxiety and Depression using Education and Skills; TAU, treatment as usual.

measures. The secondary outcome measure was the SF-12. ${ }^{17}$ Descriptions of each inventory are provided in Table $1 .^{16-19}$

\section{Intervention}

The brief CBT intervention is called Managing Anxiety and Depression using Education and Skills (MADES). It was developed by a team of experts with knowledge in depression, anxiety, and CBT principles. MADES uses a manualized approach to address the needs of patients who have CHD with preoperative depression and/or anxiety. The MADES intervention consisted of four 60-minute treatment sessions. Two clinical psychologists administered the MADES intervention over the course of the study. All treatment sessions were videotaped, and a random $20 \%$ were reviewed by the lead investigator. Videotaped reviews indicated adherence and compliance with the protocol. Table 2 provides an overview of the MADES intervention.

\section{Data Analysis}

$\chi^{2}$ and unpaired $t$ tests were performed to evaluate potential confounding group demographic and medical variables between the TAU group and the MADES group. Demographics and preoperative data collected and analyzed for each patient included age, gender, race, median household income, New York Heart Association heart failure functional class, number of affected vessels, and Comorbidity Index of Deyo and associates. ${ }^{20}$ The Comorbidity Index of Deyo and associates, adapted from the Charlson Comorbidity Index, is designed to capture comorbid conditions recorded in the inpatient setting using ICD-9-CM diagnosis and procedure codes. ${ }^{21}$ This index has been widely used in outcomes studies with administrative datasets as the principal data source. ${ }^{22}$ Length of hospital stay was assessed with a 1-way analysis of variance. The primary outcomes (BDI-II, STAI-
Trait, and SF-12) were assessed with mixed-model repeated measures analyses. Mixed models were specified with a between-subjects variable of treatment group $(1=$ intervention and $0=\mathrm{TAU})$, a within-subject variable of time $(0=$ baseline, $1=$ posttest 1 , and $2=$ posttest 2$)$, and a treatment by time interaction. Maximum likelihood determined population parameters for the fixed effects. All analyses were conducted with SPSS version 17 (SPSS, Inc, Chicago, Ill).

\section{RESULTS}

\section{Sample Characteristics}

$\chi^{2}$ and $t$ tests were used to examine potential differences between the TAU and the MADES groups. Table 3 provides descriptive statistics for the group-specific samples. In general, the 2 groups were similar on demographic and medical variables. Effect size estimates suggest that most of these differences were very small in magnitude (range of coefficients, 0.016-0.253). The only variable that was significantly different was diabetes with the TAU group having more patients with diabetes than the MADES group. This difference was moderate in magnitude (Cohen's $d=0.443$ ).

\section{Intervention Implementation}

Of the 100 patients who entered the study, 3 patients did not complete the intervention. One patient died during the 
TABLE 1. Psychometric properties and descritions of instruments

\begin{tabular}{|c|c|c|}
\hline Measures & Cronbach alpha & Description, psychometric properties \\
\hline Beck Depression Inventory-II (BDI-II) & .83 & $\begin{array}{l}\text { A } 21 \text { - item, self-report measure. Scores of } 14 \text { or greater was used to indicate } \\
\text { significant symptoms of depression. The BDI-II has strong reliability and validity } \\
\text { with medically ill patients and has been found to be sensitive to change in } \\
\text { psychotherapy trials. }{ }^{18}\end{array}$ \\
\hline State-Trait Anxiety Inventory (STAI) & .81 & $\begin{array}{l}\text { A } 20 \text {-item self-report measure of trait or dispositional anxiety. Scores of } 40 \text { or } \\
\text { greater were used to classify patients as having significant symptoms of anxiety. } \\
\text { The STAI-Trait has been validated in cardiopulmonary patients. }{ }^{19}\end{array}$ \\
\hline $\begin{array}{l}\text { Mini International Neuropsychological } \\
\text { Interview (MINI) }\end{array}$ & - & $\begin{array}{l}\text { A brief, semistructured diagnostic interview used to diagnose patients with } \\
\text { psychiatric disorders. The MINI has demonstrated adequate reliability and } \\
\text { validity for making DSM diagnoses. }{ }^{16}\end{array}$ \\
\hline Short Form-12 Health Survey (SF-12) & .79 & $\begin{array}{l}\text { The SF-12 scale is an abbreviated version of the Short Form-36 (SF-36). }{ }^{17} \text { The } \\
\text { SF-12 is a self-report inventory that measures both the physical and mental health } \\
\text { and consists of } 12 \text { questions that assess } 8 \text { different areas: physical functioning, } \\
\text { role-physical, bodily pain, general health perception, vitality, social functioning, } \\
\text { role-emotional, and mental health. Higher values on the transformed } 0 \text { to } 100 \\
\text { scale for each health domain indicate better health status. }\end{array}$ \\
\hline
\end{tabular}

DSM, Diagnostic and Statistical Manual of Mental Disorders.

hospital stay. The other 2 patients dropped out of the study ( 1 patient in each group). All the remaining 48 patients in the MADES group received all 4 sessions of the intervention. Overall, patients had a positive impression of the intervention. At the end of the intervention, patients found the intervention to be helpful in reducing symptoms of depression (mean $=7.3 ; \mathrm{SD}=1.2$; range, 5-10) and anxiety (mean $=8.3, \mathrm{SD}=0.9$, range 6-10), and they reported that they would recommend this intervention to a friend $($ mean $=7.1 ; \mathrm{SD}=0.9 ;$ range, $7-10)$.

TABLE 2. Overview of MADES therapy

\begin{tabular}{|c|c|c|c|}
\hline $\begin{array}{c}\text { Session } \\
\text { no. }\end{array}$ & $\begin{array}{c}\text { Duration } \\
\text { (min) }\end{array}$ & When & Content \\
\hline 1 & 60 & Before surgery & $\begin{array}{l}\text { Overview of study } \\
\text { Psychoeducation } \\
\text { (education about CAD, } \\
\text { surgery, depression/ } \\
\text { anxiety, etc) } \\
\text { Overview of CBT Identify } \\
\text { concerns (generate } \\
\text { problem list) }\end{array}$ \\
\hline 2 & 60 & Before surgery & $\begin{array}{l}\text { Review behavioral goals } \\
\text { Introduce cognitive strategies }\end{array}$ \\
\hline 3 & 60 & $\begin{array}{l}\text { Three days after } \\
\text { surgery }\end{array}$ & $\begin{array}{l}\text { Review cognitive strategies } \\
\text { Review cognitive distortions } \\
\text { Continue to support and } \\
\text { encourage }\end{array}$ \\
\hline 4 & 60 & $\begin{array}{l}\text { Five days after } \\
\text { surgery }\end{array}$ & $\begin{array}{l}\text { Review materials } \\
\text { Generate plan for continued } \\
\text { change } \\
\text { Continue to support and } \\
\text { encourage }\end{array}$ \\
\hline
\end{tabular}

MADES, Managing Anxiety and Depression using Education and Skills; $C A D$, coronary artery disease; $C B T$, cognitive behavioral therapy.

\section{Outcome Evaluation}

One-way analysis of variance was used to determine whether the MADES group differs significantly from the TAU group on length of hospital stay after CABG surgery. There was a significant effect of treatment on in-hospital length of stay: $F(1,95)=3.97, P=.049$.

Two-way mixed design repeated-measures analysis of variances (with independent measures on treatment and repeated measures on time) were used to assess change across baseline and postbaseline scores on the BDI-II, SF-12, and STAI-Trait inventories. Table 4 provides means and standard deviations for the outcome variables in the study. In regard to the BDI-II, it was significant within group main effect of time on BDI-II scores: $F(2,196)=6.59$, $P=.002$.

There was also a significant difference between-group effect indicating that mean BDI-II scores were significantly different between those in the TAU and MADES groups: $F(1,98)=8.69, P=.004$. There was significant interaction effect between treatment groups and time: $F(2,196)=5.31$, $P=.006$. The interaction between treatment group and time on BDI-II scores is shown in Figure 2.

STAI-Trait was significant within-group main effect of time on STAI-Trait scores: $F(2,196)=59.6, P<.001$. Between-group effect of treatment was also a significant indication that the mean STAI-Trait scores were significantly different between those in the TAU and MADES groups: $F(1,98)=17.1, P<.001$. There was a significant interaction effect between treatment groups and time: $F(2,196)=11.4, P<.001$. Figure 3 displays the interaction between treatment group and time on STAI-Trait scores.

Moreover, the SF-12, was significantly different in the within-group main effect of time on SF-12 scores: 
TABLE 3. Descriptive statistics for TAU and MADES therapy groups

\begin{tabular}{|c|c|c|c|c|}
\hline Variable & $\begin{array}{c}\text { TAU } \\
(n=49)\end{array}$ & $\begin{array}{l}\text { MADES } \\
(n=48)\end{array}$ & P value & Effect size* \\
\hline Age (y) & $64.2(\mathrm{SD}=11.9)$ & $62.8(\mathrm{SD}=11.8)$ & .562 & .118 \\
\hline Gender (male) & $79.6 \%$ & $77.1 \%$ & & \\
\hline Race & & & .655 & .092 \\
\hline White & $38(77.6 \%)$ & $39(81.2 \%)$ & & \\
\hline Other & $11(22.4 \%)$ & $9(18.8 \%)$ & & \\
\hline Median household income & & & .479 & .144 \\
\hline$\$ 1-\$ 44,900$ & $27(55.1 \%)$ & $23(47.9 \%)$ & & \\
\hline$\$ 45,000$ or more & $22(44.9 \%)$ & $25(52.1 \%)$ & & \\
\hline NYHA class & $2.88(\mathrm{SD}=0.4)$ & $2.92(\mathrm{SD}=0.4)$ & .624 & .100 \\
\hline No. of affected vessels & $2.96(\mathrm{SD}=0.6)$ & $3.10(\mathrm{SD}=0.5)$ & .215 & .253 \\
\hline Arrhythmias & $12(24.4 \%)$ & $10(20.1 \%)$ & .662 & .043 \\
\hline CPB time (min) & $84.6(\mathrm{SD}=26.2)$ & $91.6(\mathrm{SD}=31.4)$ & .236 & .242 \\
\hline Psychiatric medications & $8(16.3 \%)$ & $12(24.4 \%)$ & .291 & .107 \\
\hline Preoperative beta blockers & $38(77.5 \%)$ & $41(85.4 \%)$ & .319 & .101 \\
\hline Duration of ICU stay (h) & $46.3(\mathrm{SD}=23.6)$ & $48.9(\mathrm{SD}=20.3)$ & .562 & .118 \\
\hline Left ventricular ejection fraction $(\%)$ & $49.1(\mathrm{SD}=12.9)$ & $52.3(\mathrm{SD}=15.3)$ & .267 & .226 \\
\hline Deyo et $\mathrm{al}^{20}$ index & $1.31(\mathrm{SD}=1.10)$ & $1.29(\mathrm{SD}=1.08)$ & .928 & .018 \\
\hline Myocardial infarction & $17(34.7 \%)$ & $17(35.4 \%)$ & .941 & .016 \\
\hline Congestive heart failure & $10(20.4) \%$ & $11(22.9 \%)$ & .764 & .061 \\
\hline Peripheral vascular disease & $4(8.2 \%)$ & $3(6.4 \%)$ & $.716 \dagger$ & .074 \\
\hline Cerebrovascular disease & $3(6.1 \%)$ & $2(4.2 \%)$ & $.989 \dagger$ & .089 \\
\hline Dementia & $0(0.0 \%)$ & $0(0.0 \%)$ & - & - \\
\hline COPD & $8(16.3 \%)$ & $6(12.5 \%)$ & .592 & .101 \\
\hline Rheumatologic disease & $1(2.0 \%)$ & $0(0.0 \%)$ & $.320 \dagger$ & .203 \\
\hline Peptic ulcer disease & $0(0.0 \%)$ & $0(0.0 \%)$ & - & - \\
\hline Mild liver disease & $0(0.0 \%)$ & $0(0.0 \%)$ & - & - \\
\hline Diabetes & $20(40.8 \%)$ & $10(20.8 \%)$ & .033 & .443 \\
\hline Diabetes chronic complications & $2(4.1 \%)$ & $1(2.1 \%)$ & $.570 \dagger$ & .116 \\
\hline Hemiplegia or paraplegia & $0(0.0 \%)$ & $0(0.0 \%)$ & - & - \\
\hline Renal disease & $1(2.0 \%)$ & $0(0.0 \%)$ & $.320 \dagger$ & .203 \\
\hline Malignancy & $0(0.0 \%)$ & $0(0.0 \%)$ & - & - \\
\hline Moderate/severe liver disease & $0(0.0 \%)$ & $0(0.0 \%)$ & - & - \\
\hline Metastatic solid tumor & $0(0.0 \%)$ & $0(0.0 \%)$ & - & - \\
\hline AIDS/HIV & $0(0.0 \%)$ & $0(0.0 \%)$ & - & - \\
\hline
\end{tabular}

TAU, Treatment as usual; MADES, Managing Anxiety and Depression using Education and Skills; SD, standard deviation; NYHA, New York Heart Association Classification; $C P B$, cardiopulmonary bypass; $I C U$, intensive care unit; $C O P D$, chronic obstructive pulmonary disease; $A I D S / H I V$, acquired immunodeficiency disease/human immunodeficiency virus. *Cohen's $d$ statistics were calculated using pooled variance and adjusted for unequal sample sizes for continuous variables and phi coefficients were calculated for dichotomous variables. $†$ Fisher exact test calculated for significance testing.

$F(1,98)=68.8, P<.001$. There was no significant effect of treatment group indicating that mean SF-12 scores for those in the MADES and TAU groups were in general the same: $F(1,98)=3.22, P=.076$. Also, there was no significant interaction effect between treatment groups and time: $F(1,98)=.780, P=.379$. Figure 4 displays the interaction between treatment group and time on SF-12 scores.

TABLE 4. Means and standard deviations for outcome variables

\begin{tabular}{|c|c|c|c|c|c|c|c|c|c|c|c|c|}
\hline & \multicolumn{6}{|c|}{ MADES } & \multicolumn{6}{|c|}{ TAU } \\
\hline & \multicolumn{2}{|c|}{ PRE1 } & \multicolumn{2}{|c|}{ POST1 } & \multicolumn{2}{|c|}{ POST2 } & \multicolumn{2}{|c|}{ PRE1 } & \multicolumn{2}{|c|}{ POST1 } & \multicolumn{2}{|c|}{ POST2 } \\
\hline & $\mathbf{M}$ & SD & $\mathbf{M}$ & SD & M & SD & M & SD & $\mathbf{M}$ & SD & M & SD \\
\hline STAI-Trait & 55.8 & 11.8 & 36.6 & 10.9 & 40.6 & 12.1 & 55.4 & 11.2 & 49.0 & 7.4 & 45.6 & 7.7 \\
\hline BDI-II & 23.0 & 6.6 & 15.9 & 5.1 & 19.2 & 6.7 & 22.4 & 6.2 & 23.4 & 11.6 & 22.5 & 10.7 \\
\hline SF-12 & 37.1 & 9.7 & - & - & 48.6 & 11.1 & 38.3 & 9.9 & - & - & 52.6 & 11.3 \\
\hline LOS & - & - & 7.9 & 2.6 & - & - & - & - & 9.2 & 3.5 & - & - \\
\hline
\end{tabular}

MADES, Managing Anxiety and Depression using Education and Skills; TAU, treatment as usual; PRE1, baseline; POST1, in-hospital follow-up; POST2, 3 to 4 weeks of followup; $M$, mean; SD, standard deviation; STAI, State-Trait Anxiety Inventory; BDI-II, Beck Depression Inventory-II; SF-12, Short Form-12 Health Survey scale; LOS, length of inpatient stay. 


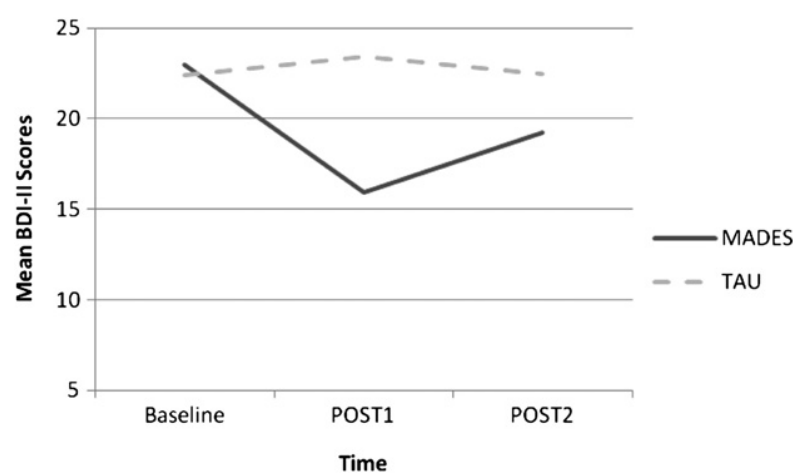

FIGURE 2. Time by treatment group interaction on Beck Depression Inventory-II (BDI-II) scores. MADES, Managing Anxiety and Depression using Education and Skills; TAU, treatment as usual.

\section{DISCUSSION}

CBT and psychoeducation approaches are considered among the most robust and safe evidenced-based interventions to treat depression and anxiety in psychology and psychiatry, and they are among the most widely implemented treatments. CBT for anxiety and depression disorders usually involves a combination of behavioral techniques such as muscle relaxation and cognitive-restructuring techniques aimed at reducing distorted cognitions and information processing biases. CBT has been shown repeatedly to be equally effective to antidepressant medications in reducing depressive symptoms. ${ }^{23} \mathrm{~A}$ distinct advantage of CBT over medications is that they generally produce more longlasting improvement with very low relapse rates. ${ }^{23}$

Moreover, despite the high prevalence of depression and anxiety before CABG surgery, they are underrecognized and undertreated in the medical populations in general and among cardiovascular patients in particular. ${ }^{8}$ Undertreatment of these disorders affects the quality of life, quality of medical care, and cost of health care utilization. ${ }^{24}$ This might be largely due to the difficulty in advocating for the recognition and treatment of an additional condition in patients hospitalized for cardiac disease, as well as over-

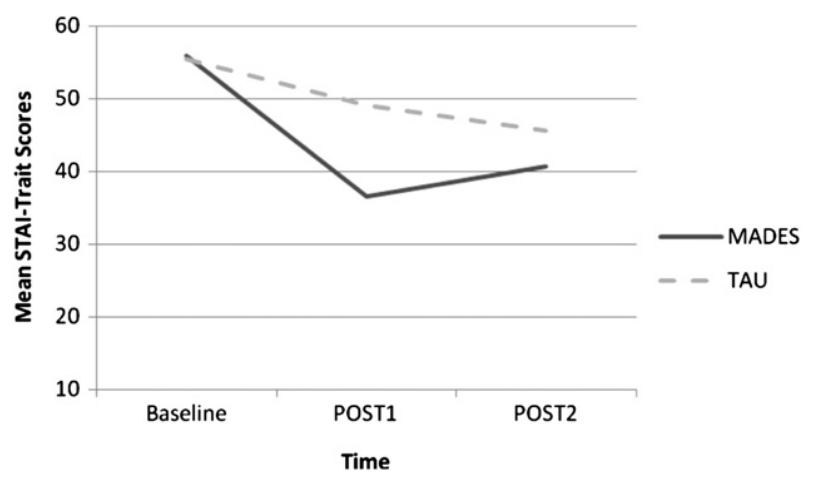

FIGURE 3. Time by treatment group interaction on State-Trait Anxiety Inventory-Trait (STAI-Trait) scores. MADES, Managing Anxiety and Depression using Education and Skills; TAU, treatment as usual.

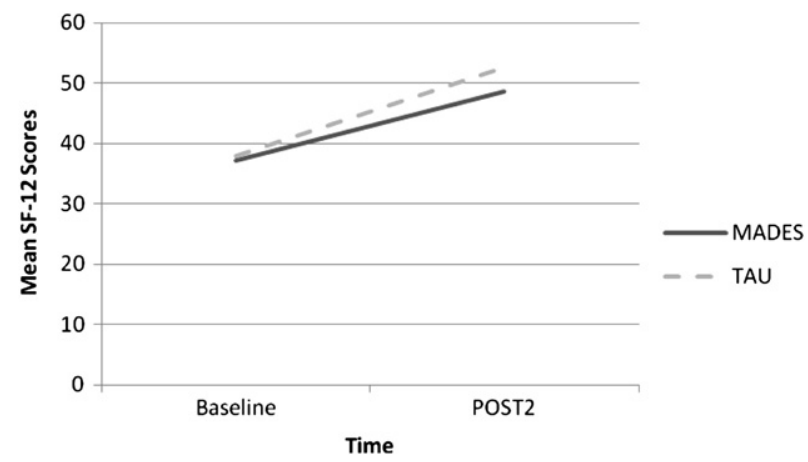

FIGURE 4. Time by treatment group interaction on Short Form-12 Health Survey ( $S F-12$ ) scores. MADES, Managing Anxiety and Depression using Education and Skills; TAU, treatment as usual.

lap in symptoms (such as fatigue, decreased appetite, worry, sleep difficulties, and changes in sexual desire). ${ }^{8}$ Nevertheless, even with appropriate training in assessment of depression, treating depression with traditional CBT approaches is a challenge. Traditional CBT approaches usually require a longer period of time. This is particularly problematic in surgical patients inasmuch as the period between informing patients of the indication for CABG surgery to the actual surgery is usually limited. Thus, we developed and tested a brief, tailored CBT intervention called Managing Anxiety and Depression using Education and Skills (MADES).

The results of the study suggested that the MADES intervention, when initiated preoperatively, led to improvements in both depressive and anxiety symptoms in patients undergoing CABG surgery, as well as improving patients' quality of life. It has also been shown that the intervention is both feasible and acceptable for patients undergoing CABG surgery. Moreover, MADES treatment reduced in-hospital length of stay.

The low dropout rate was a reflection of the feasibility and acceptability of the study and may also be an indirect reflection of its efficacy and lack of adverse effects associated with it. Of the 50 patients who were assigned to the MADES group, only 1 patient dropped out of the study and did not complete the intervention. In the TAU group, there was also 1 patient who asked to be dropped from the study. Moreover, on the basis of self-reports, patients affirmed that the intervention was helpful in reducing depression and anxiety and that they would recommend the intervention to a friend.

Patients in the TAU group had higher BDI-II scores (indicating higher depressive symptomatology) on their posttest compared with their pretest assessment. On the contrary, patients in the MADES group had lower BDI-II scores on their posttest compared with their pretest assessment. The finding that BDI-II scores actually increased on the posttest assessment in the TAU group suggests that if depressive symptoms go untreated, these symptoms could actually be exacerbated after surgery. In addition, 
improvements in anxiety symptoms were reflected by significant improvement in the STAI-Trait scores showing significant interaction effect between treatment groups and time.

Patients in the MADES group have lower length of hospital stay than do patients in the TAU group. Specifically, patients in the TAU group, on average, stayed an extra day longer in the hospital than did patients in the MADES. As the cost of inpatient hospitalization continues to increase, this finding has both clinical and practical implications. For instance, the total cost associated with the surgery and in-hospital stay is about $\$ 38,000$ (about $\$ 4000$ per hospital day). ${ }^{25}$ Reducing hospitalization even by 1 day will decrease cost by several thousand dollars per patient. This will also reduce use of resources such as hospital staff, equipment, and beds, which makes them available for other patients in need of care.

Despite the positive findings of the study, there are several limitations to consider. This study did not use multiple interviewers to assess diagnostic reliability. However, diagnoses were made by an experienced clinician using the MINI. Second, the majority of patients in both the MADES and TAU groups had comorbid diagnoses. Thus, some caution is warranted in the interpretation of the effect of the treatment of depression or anxiety. On the other hand, this limitation can also be viewed as increasing the external validity and generalizablity of the study to real life clinical settings, given that in clinical settings, comorbidity of psychiatric disorders is more common than single morbidity. ${ }^{26}$ Third, no information was obtained regarding the differences in pain medications between the TAU and the MADES groups at the time that postoperative testing was conducted, which could influence the results of the study (ie, in-hospital and 3-4 weeks of follow-up). Fourth, inasmuch as the study was not able to blind the surgeons to which group the patients were assigned to, experimenter bias may be a threat to the internal validity of this study. Fifth, this study was conducted with a population of veterans; thus, the results of the study cannot be generalized to the civilian population. Finally, although pain medications were assessed at the beginning of the study, there were no pain medication assessments after surgery. Thus, the results of the treatment may be due to an interactional effect between pain medications and the MADES intervention.

\section{References}

1. Centers for Disease Control and Prevention (CDC). Web-based Injury Statistics Query and Reporting System (WISQARS). (2005). National Center for Injury Prevention and Control, CDC (producer). Available at: http://www.cdc.gov/ ncipc/wisqar/default.htm.

2. Blumenthal JA, Newman M, Babyak MA, Lett HS, Mark D. Depression as a risk factor for mortality after coronary artery bypass surgery. Lancet. 2003;362: 604-9.
3. Borowicz L, Royall R, Grega M, Selnes O, Lyketsos C, McKhann G. Depression and cardiac morbidity 5 years after coronary artery bypass surgery. Psychosom Med. 2002;43:464-71.

4. Burg MM, Benedetto C, Rosenberg R, Soufer R. Presurgical depression predicts medical morbidity 6 months after coronary artery bypass graft surgery. Psychosom Med. 2003;65:111-8.

5. Oxlad M, Stubberfield J, Stuklis R, Edwards J, Wade TD. Psychological risk factors for cardiac-related hospital readmission within 6 months of coronary artery bypass graft surgery. J Psychosom Res. 2006;61:775-81.

6. Tully PJ, Baker RA, Knight JL. Anxiety and depression as risk factors for mortality after coronary artery bypass surgery. J Psychosom Res. 2008;64 285-90.

7. Freedland KE, Skala JA, Carney RM, Rubin EH, Lustman PJ, DavilaRoman VG, et al. Treatment of depression after coronary artery bypass surgery: a randomized controlled trial. Arch Gen Psychiatry. 2009;66:387-96.

8. Rumsfeld JS, Ho M. Depression and cardiovascular disease. Circulation. 2005; 111:250-3.

9. Freedland KE, Skala JA, Carney RM, Rubin EH, Lustman PJ, DavilaRoman VG, et al. Treatment of depression after coronary artery bypass surgery: a randomized controlled trial. Arch Gen Psychiatry. 2009;66:387-96.

10. Rollman BL, Belnap BH, LeMenager MS, Mazumdar S, Houck PR, Counihan PJ, et al. Telephone-delivered collaborative care for treating postCABG depression: a randomized controlled trial. JAMA. 2009;302:2095-103.

11. Lie I, Arnesen H, Sandvik L, Hamilton G, Bunch EH. Effects of a home-based intervention program on anxiety and depression 6 months after coronary artery bypass grafting: a randomized controlled trial. J Psychosom Res. 2007;62:411-8.

12. Levin A. Depression, heart disease: links remains elusive. Psychiatry News. 2005;40:33-5.

13. Berkman LF, Blumenthal J, Burg M, Carney RM, Catellier D, Cowan MJ, et al Effects of treating depression and low perceived social support on clinical events after myocardial infarction: the Enhancing Recovery in Coronary Heart Disease Patients (ENRICHD) Randomized Trial. JAMA. 2003;289:3106-16.

14. Beck AT, Steer RA, Brown GK. Manual for the Beck Depression Inventory. 2nd edition. San Antonio (TX): The Psychological Corporation; 1996.

15. Speilberger CD, Gorsuch RL, Lushene R, Vagg PR, Jacobs GA. Manual for the State-Trait Anxiety Inventory. Palo Alto (CA): Consulting Psychologist; 1970.

16. Sheehan DV, Lecrubier Y, Sheehan KH, Amorim P, Janavs J, Weiller E, et al. The Mini-International Neuropsychiatric Interview (M.I.N.I.): the development and validation of a structured diagnostic psychiatric interview for DSM-IV and ICD-10. J Clin Psychiatry. 1998;59:22-33.

17. Ware JE, Snow KK, Kosinski M, Gandek B. SF-36 Health Survey: manual and interpretation guide. Boston: The Health Institute; 1993.

18. Stanley MA, Wilson NL, Novy D, Rhoades H, Wagener P, Greisinger AJ, et al Cognitive behavior therapy for generalized anxiety disorder among older adults in primary care: a randomized clinical trial. JAMA. 2009;301:1460-7.

19. Kawachi I, Colditz GA, Ascherio A, Rimm EB, Giovannucci E, Stampfer MJ, et al. Prospective study of phobic anxiety and risk of coronary heart disease in men. Circulation. 1994;89:1992-7.

20. Deyo RA, Cherkin DC, Ciol MA. Adapting a clinical comorbidity index for use with ICD-9-CM administrative databases. J Clin Epidemiol. 1992;45:613-9.

21. Charlson ME, Pompei P, Ales KL, MacKenzie CR. A new method of classifying prognostic comorbidity in longitudinal studies: development and validation. J Chronic Dis. 1987:40:373-83.

22. Needham D, Scales D, Laupacis A, Pronovost P. A systematic review of the Charlson comorbidity index using Canadian administrative databases: a perspective on risk adjustment in critical care research. $J$ Crit Care. 2005;20:12-9.

23. DeRubeis RJ, Gelfand LA, Tang TZ, Simons AD. Medications versus cognitive behavior therapy for severely depressed outpatients: meta-analysis of four randomized comparisons. Am J Psychiatry. 1999;156:1007-13.

24. Ford JD, Trestman RL, Tennen H, Allen S. Relationship of anxiety, depression and alcohol use disorders to persistent high utilization and potentially problematic under-utilization of primary medical care. Soc Sci Med. 2005;61: 1618-25.

25. Chu D, Bakaeen F, Dao T, LeMaire SA, Coselli J, Huh J. On-pump versus offpump coronary bypass grafting in a cohort of 63,000 patients. Ann Thorac Surg. 2009;87:1820-7.

26. Kaplan HI, Sadock BJ. Synopsis of psychiatry. 8th edition. Baltimore: Williams \& Wilkins; 1998. 